\title{
Essential oils of Baccharis trinervis (Asteraceae) from Costa Rica
}

\section{Carlos Chaverri \& José F. Cicció*}

Escuela de Química and Centro de Investigaciones en Productos Naturales (CIPRONA), Universidad de Costa Rica, 11501-2060, San José, Costa Rica; carloschaverri@yahoo.com, jfciccio@gmail.com

\author{
Received 11-V-2017. Corrected 20-VII-2017. Accepted 23-VIII-2017.
}

\begin{abstract}
Baccharis is an Asteraceae genus of flowering plants, which has about 340 to 400 species, ranging from the Southern United States to the Southern extreme of Argentina and Chile through Central America and the Caribbean regions. The species Baccharis trinervis is a native shrub from Mexico, Central America and throughout South America. In Costa Rica, this species is commonly known as alcotán and the fresh leaves are used as a poultice on wounds and ulcers. The objective of the present research was to characterize the chemical composition of seven hydrodistilled essential oils of diverse morphological parts of $B$. trinervis. For this, samples were obtained from three locations in Costa Rica and standard laboratory analyses were followed. The essential oils were analyzed by capillary gas chromatography-flame ionization detector (GC-FID) and gas chromatography-mass spectrometry (GC-MS) using the retention indices on a $5 \%$ phenyl/dimethylpolysiloxane fused silica column in addition to mass spectral fragmentation patterns, which allowed the identification of 268 compounds. The essential oils consisted mainly of terpenoids (92.3 to $97.8 \%$ ). The major constituents from the leaf oils were caryophyllene oxide (0.1-22.5\%), viridiflorol (8.8-21.0\%), germacrene D (0.5-19.1\%), germacrene B (0.2-16.0\%), $\beta$-caryophyllene (3.5-9.1\%), spathulenol (0.1-8.3\%), $\delta$-3-carene (2.0-6.8 \%), and $\alpha$-pinene (2.5-5.9\%). The flower oil consisted mainly of globulol (0-24\%), $\beta$-caryophyllene $(9.5-17.1 \%)$, cismuurola-4(14), 5-diene (traces-13.7 \%), germacrene D (4.3-9.9\%), bicyclogermacrene (5.9-8.3 \%), ar-curcumene (0-8.0 \%), spathulenol (4.3-4.8\%), caryophyllene oxide (3.1-4.7\%), and viridiflorol (0.3-4.7\%). The major components of the branch oil were germacrene B (1.4-18.7 \%), germacrene D (14.7-15.6 \%), $\beta$-caryophyllene (10.1-12.4\%), viridiflorol (0-11.5\%), globulol (0.6-11.3\%), $\delta$-3-carene (4.1-8.1\%), $\beta$-phellandrene (1.5-6.5\%), and bicyclogermacrene (3.6-4.9\%). The essential oil composition differed markedly from that of previously studied oils of plants growing in Brazil, which contain two characteristic stereoisomeric methyl dec-2-en4,6-diynoate compounds not detected in this study. This is the first report about the chemical composition of the essential oils obtained from this species growing wild in Costa Rica. Rev. Biol. Trop. 65 (4): 1307-1321. Epub 2017 December 01.
\end{abstract}

Key words: Baccharis trinervis, Asteraceae, essential oils, terpenoids, GC-MS, Costa Rica.

Baccharis L. is one of the largest genera within Asteraceae family (tribe Astereae, sub-tribe Baccharidinae) including herbaceous perennials, vines, shrubs and trees. This genus includes ca. 340 to 400 species ranging from the Southern United States to the Southern extreme of Argentina and Chile through Central America and the Caribbean basin (Heiden, Andrade-Baumgratz, \& Esteves, 2012). Some of the species are economically important as medicinals -used mainly as infusions and decoctions- for treat stomach and liver ailments, inflammations, anemia, diabetes and prostate diseases (Verdi, Brighente, \& Pizzolatti, 2005). The vassoura essential oil (Baccharis dracunculifolia DC.) and carqueja oil [B. genistelloides (Lam.) Pers.] are used in perfume industry (Ferracini et al., 1995).

Baccharis trinervis Pers. [=Pseudobaccharis trinervis (Pers.) V. M. Badillo] is a common shrub or sub-scandent shrub in the Pacific slope of Costa Rica, with hairless ribbed stems 
and oblong-elliptic or lanceolate-elliptic alternate leaves, 3 to $10 \mathrm{~cm}$ long, 1.5 to $3.5 \mathrm{~cm}$ wide, hard and rough, with three prominent, longitudinal veins, puberulent to pilose on both sides. Flowers are greenish-white disposed in terminal panicles (León \& Poveda, 2000) situated within or overtopping the foliage. This plant has a wide distribution, from Mexico, Central America and throughout South America. In Costa Rica it is distributed over the country, from lowlands to $1900 \mathrm{~m}$ of elevation, in both dry and wet forests. This plant is commonly known as alcotán, and in the Northwestern Guanacaste Province it is known as jalapatrás and rastrapo (León \& Poveda, 2000). In Costa Rica, the fresh leaves are used as a poultice on wounds and ulcers (Pittier, 1978) and the alcoholic extracts of roots are said to be used as remedy for snakebites (Núñez-Meléndez, 1978). In Honduras, the extracted juice of the leaf, and root decoctions, are taken as a remedy for kidney troubles, pain and inflammations, as a febrifuge, for halting diarrhea, and against rheumatic pains (House et al., 1995). It has also been used in various liver diseases, as a purgative, antiseptic, digestive and diuretic, in rectal washes against hemorrhoids and as a remedy for typhoid fever (Ramírez-Cárdenas, IsazaMejía, \& Pérez-Cárdenas, 2013).

Many phytochemical investigations have been performed on plants of the Baccharis genus and are characterized by the occurrence of flavonoids, diterpenoids (the most prominent classes are clerodanes and labdanes, and with some frequency, kauranes), triterpenes, phenolic compounds, and particularly important is the occurrence of essential oils (Verdi et al., 2005; Abad \& Bermejo, 2007; Ramos Campos et al., 2016). From the aerial parts of Baccharis trinervis, Bohlmann and Zdero (1970) reported the presence of matricaria ester, and three C-17 esters with an ene-diyne-diene-structure. From air-dried aerial parts (from Brazil) were obtained the terpenoids phytene, caryophyllene, germacrene $\mathrm{D}$, bicyclogermacrene, $\alpha$ - and $\gamma$-humulene, squalene, lupeyl acetate, lupeol, $\beta$-amyrin and the diacetylenic compound lachnophyllum ester (Bohlmann, Kramp, Grenz,
Robinson, \& King, 1981), whereas the study of Kuroyanagi, Uchida, Ueno, Satake, and Shimomura (1993) afforded 24 neo-clerodane type diterpenes, many of them new. Also, Herrera, Rosas-Romero, Crescente, Acosta, and Pekerar (1996) reported several 5-hydroxy7-methoxyflavones from leaves, and Sharp et al. (2001) reported three 6-oxigenated flavones from branches.

Regarding biological activities attributed to this species there are some reports about antioxidant activity of the ethanolic extract (Heras et al., 1998), antiviral activity against herpes simplex type I (HSV-1), vesicular stomatitis virus (VSV-1) and poliovirus type I (Abad, Bermejo, Sánchez-Palomino, Chiriboga, \& Carrasco, 1999), anti-HIV activity in macrophages (Sánchez-Palomino et al., 2002), antimicrobial activity (Albuquerque et al., 2004), and antioxidant, antifungal and hemolytic activities of the essential oil (Sobrinho et al., 2016).

In relation to the essential oil composition of the aerial parts of this species, there are only three previous reports: two studies from material collected in the state of Ceará, Northeast Brazil (Albuquerque et al., 2004; Sobrinho et al., 2016), and another from the state of Mérida, Venezuela (Rojas et al., 2008).

The objective of the present research was the characterization of the chemical composition of seven different samples of essential oils from diverse morphological parts of $B$. trinervis collected in three different locations in central Costa Rica. To the best of our knowledge, no previous chemical work on $B$. trinervis from Costa Rica has been reported.

\section{MATERIALS AND METHODS}

Plant material: The aerial parts of Baccharis trinervis were collected from three localities of Costa Rica: Miramar, Montes de Oro mountains (west central Pacific), Province of Puntarenas $\left(10^{\circ} 06^{\prime} 12.79^{\prime}{ }^{\prime} \mathrm{N}-84^{\circ} 42^{\prime} 26.09^{\prime \prime} \mathrm{W}\right.$, at an elevation of $750 \mathrm{~m}$ ), on April 2009, during the flowering stage; in the University of Costa Rica Campus, San Pedro de Montes de Oca, Province of San José (9॰56'14.60” N - 8402'51.51” W, 
at 1210 m elevation), on February 2009, during vegetative stage, and Pavas, Province of San

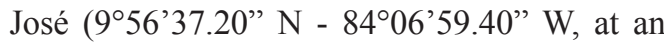
elevation of $1093 \mathrm{~m}$ ) on November 2015, during the flowering stage of the plant. A voucher specimen has been deposited in the Herbarium of the University of Costa Rica at the School of Biology (USJ 93916).

Isolation of the essential oils: Plant materials were hydrodistilled at atmospheric pressure, for $3 \mathrm{~h}$, using a Clevenger-type apparatus. The distilled oils were collected and dried over anhydrous sodium sulfate, filtered and stored at $0-10{ }^{\circ} \mathrm{C}$ in the dark, for further analysis. The essential oil yields $(\mathrm{v} / \mathrm{w})$ from diverse morphological parts from three localities in central Costa Rica were: Miramar sample, $0.32 \%$ (leaf), $0.04 \%$ (flower); San Pedro sample, $0.35 \%$ (leaf), $0.03 \%$ (branch); and Pavas sample, $0.23 \%$ (leaf), $0.06 \%$ (flower), and $0.05 \%$ (branch).

Gas chromatographic (GC-FID) analyses: The oils of Baccharis trinervis were analyzed using a Shimadzu GC-2014 gas chromatograph. The data were obtained on a $5 \%$ phenyl/dimethylpolysiloxane column (30 m x $0.25 \mathrm{~mm}$; film thickness $0.25 \mu \mathrm{m}$ ), (MDN-5S, Supelco), with a Shimadzu GCsolution Chromatography Data System, Shimadzu GC Solution, Chromatography Data System, software version 2.3. The experimental conditions were: carrier gas $\mathrm{N}_{2}$, flow $1.0 \mathrm{~mL} / \mathrm{min}$; oven temperature program: 60 to $280{ }^{\circ} \mathrm{C}$ at $3{ }^{\circ} \mathrm{C} / \mathrm{min}, 280{ }^{\circ} \mathrm{C}$ (2 min); sample injection port temperature $250{ }^{\circ} \mathrm{C}$; detector temperature $280{ }^{\circ} \mathrm{C}$; split 1:60.

Gas chromatography-mass spectrometry (GC-MS): The analyses were performed using a Shimadzu GC-17A gas chromatograph coupled with a GCMS-QP5000 apparatus and CLASS 5000 software with Wiley 139 and NIST computer databases. The data were obtained on a MDN-5S column (30 m x $0.25 \mathrm{~mm}$ ), coated with $5 \%$ phenyl/dimethylpolysiloxane (film thickness $0.25 \mu \mathrm{m}$ ). Experimental conditions were: carrier gas $\mathrm{He}$, flow
$1.0 \mathrm{~mL} / \mathrm{min}$; oven temperature program: 60 to $280{ }^{\circ} \mathrm{C}$ at $3{ }^{\circ} \mathrm{C} / \mathrm{min}$; sample injection port temperature $250{ }^{\circ} \mathrm{C}$; detector temperature $260{ }^{\circ} \mathrm{C}$; ionization voltage: $70 \mathrm{eV}$; ionization current $60 \mu \mathrm{A}$; scanning speed $0.5 \mathrm{~s}$ over 38 to $400 \mathrm{amu}$ range; split 1:70.

Compound identification and quantification: The components of the oils were identified by comparison of their linear retention indices which were calculated in relation to a homologous series of $n$-alkanes, on $5 \%$ phenyl/ dimethylpolysiloxane type column (van den Dool \& Kratz, 1963), and by comparison of their mass spectral fragmentation patterns with those published in the literature (Adams, 2007) or those of our own database. To obtain the retention indices for each peak, $0.1 \mu \mathrm{L}$ of $n$-alkane mixture (Sigma, $\mathrm{C}_{8}$ to $\mathrm{C}_{32}$ standard mixture) was injected under the same experimental conditions reported above. Integration of the total chromatogram (GC-FID), expressed as area percent, has been used to obtain quantitative compositional data without FID response factor correction.

\section{RESULTS}

The essential oils from different parts of Baccharis trinervis from Costa Rica presented a very complex chemical profile. The compounds identified, their experimental retention indices (RI) determined in relation to a homologous series of linear alkanes $\left(\mathrm{C}_{8}\right.$ to $\mathrm{C}_{32}$ ), their relative percentage concentrations, and the method used for their identification are presented in table 1 . The constituents are listed in order of elution on a MDN-5S column and for comparison purposes, previously published values of the retention indices are included (Lit. RI). Compounds identified in this study and previously reported in the Venezuelan and Brazilian essential oil samples are indicated by bullets and/or asterisks.

Baccharis trinervis gave essential oils which were predominantly terpenoid in nature with a few aliphatic and aromatic compounds as minor and trace constituents. In table 2, 
TABLE 1

Chemical and percentage composition of oils of Baccharis trinervis from three locations in Costa Rica

\begin{tabular}{|c|c|c|c|c|c|c|c|c|c|c|c|}
\hline \multirow{2}{*}{ Compound $^{\mathrm{a}}$} & \multirow{2}{*}{$\mathrm{RI}^{\mathrm{b}}$} & \multirow{2}{*}{ Lit. $\mathrm{RI}^{\mathrm{c}}$} & \multirow{2}{*}{ Class $^{\mathrm{d}}$} & \multicolumn{2}{|c|}{ Miramar } & \multicolumn{2}{|c|}{ San Pedro } & \multicolumn{3}{|c|}{ Pavas } & \multirow{2}{*}{$\begin{array}{l}\text { Ident. } \\
\text { methode }^{\mathrm{e}}\end{array}$} \\
\hline & & & & Leaf & Flower & Leaf & Branch & Leaf & Flower & Branch & \\
\hline Hexane & 623 & 623 & A & $\mathrm{t}$ & & & & & & & $1,2,3$ \\
\hline 3-Methylbutanal & 655 & 658 & $\mathrm{~A}$ & $\mathrm{t}$ & & & & & & & 1,2 \\
\hline 2-Ethylfuran & 702 & 702 & Misc. & $\mathrm{t}$ & & & & & & & 1,2 \\
\hline Pentanal & 704 & 704 & A & & & $\mathrm{t}$ & 0.1 & & & & 1,2 \\
\hline 4-Methylcyclohexene & 740 & 740 & $\mathrm{~A}$ & & & $\mathrm{t}$ & & & & & 1,2 \\
\hline Toluene & 768 & $770^{\mathrm{f}}$ & $\mathrm{B}$ & $\mathrm{t}$ & & & & & & & 1,2 \\
\hline Octane & 800 & 800 & $\mathrm{~A}$ & $\mathrm{t}$ & & & & & & & $1,2,3$ \\
\hline Hexanal & 801 & 801 & $\mathrm{~A}$ & & $\mathrm{t}$ & & $\mathrm{t}$ & & & & 1,2 \\
\hline (E)-Hex-2-enal & 852 & 846 & $\mathrm{~A}$ & $\mathrm{t}$ & $\mathrm{t}$ & $\mathrm{t}$ & $\mathrm{t}$ & 0.1 & & 0.1 & 1,2 \\
\hline (Z)-Salvene & 846 & 847 & M & $\mathrm{t}$ & & & & & & & 1,2 \\
\hline (E)-Hex-2-en-1-ol & 859 & 854 & $\mathrm{~A}$ & & & 0.1 & & 0.2 & & 0.1 & 1,2 \\
\hline Hexan-1-ol & 864 & 863 & A & & $\mathrm{t}$ & 0.1 & & $\mathrm{t}$ & & $\mathrm{t}$ & 1,2 \\
\hline 2-Butylfuran & 883 & $885^{\mathrm{g}}$ & Misc. & & $\mathrm{t}$ & & & & & & 1,2 \\
\hline Heptan-2-one & 888 & 889 & A & & $\mathrm{t}$ & & & & & & 1,2 \\
\hline (Z)-Hept-4-enal & 897 & 898 & $\mathrm{~A}$ & & $\mathrm{t}$ & & & & & & 1,2 \\
\hline Heptanal & 901 & 901 & $\mathrm{~A}$ & $\mathrm{t}$ & 0.1 & $\mathrm{t}$ & $\mathrm{t}$ & $\mathrm{t}$ & & $\mathrm{t}$ & 1,2 \\
\hline$\alpha$-Thujene*• & 923 & 924 & M & 1.0 & 0.3 & 1.7 & 0.3 & 1.6 & 0.1 & 0.3 & 1,2 \\
\hline$\alpha$-Pinene*• & 930 & 932 & M & 3.9 & 1.0 & 2.5 & 0.6 & 5.9 & 0.3 & 1.9 & $1,2,3$ \\
\hline Camphene*• & 946 & 946 & M & 0.1 & $\mathrm{t}$ & 0.1 & & 0.1 & & $\mathrm{t}$ & 1,2 \\
\hline Thuja-2,4(10)-diene & 952 & 953 & M & $\mathrm{t}$ & & & & & & & 1,2 \\
\hline Benzaldehyde & 959 & 952 & $\mathrm{~B}$ & $\mathrm{t}$ & $\mathrm{t}$ & $\mathrm{t}$ & & & & & 1,2 \\
\hline Sabinene*• & 970 & 969 & M & 0.8 & 0.3 & 0.4 & 0.2 & 1.6 & 0.3 & 0.3 & 1,2 \\
\hline$\beta$-Pinene*• & 978 & 974 & M & 1.6 & 1.0 & 1.3 & 0.8 & 2.5 & 0.4 & 2.1 & $1,2,3$ \\
\hline 6-Methylhept-5-en-2-one & 982 & 981 & $\mathrm{~A}$ & $\mathrm{t}$ & & & & $\mathrm{t}$ & & & 1,2 \\
\hline Myrcene*• & 986 & 988 & M & 0.2 & 0.3 & 0.8 & 0.5 & 1.3 & 0.1 & 1.4 & 1,2 \\
\hline Dehydro-1,8-cineole & 988 & 988 & M & 0.1 & & & & & & & 1,2 \\
\hline Mesitylene & 995 & 995 & $\mathrm{~B}$ & & & & & $\mathrm{t}$ & & & 1,2 \\
\hline Decane & 1000 & 1000 & A & & $\mathrm{t}$ & & & & $\mathrm{t}$ & & $1,2,3$ \\
\hline$\delta$-2-Carene & 1000 & 1001 & M & $\mathrm{t}$ & $\mathrm{t}$ & $\mathrm{t}$ & & $\mathrm{t}$ & $\mathrm{t}$ & & 1,2 \\
\hline$\alpha$-Phellandrene* & 1002 & 1002 & M & & & & & 0.1 & & & 1,2 \\
\hline$p$-Mentha-1(7)-8-diene & 1003 & 1003 & M & $\mathrm{t}$ & & & & & & & 1,2 \\
\hline (Z)-Hex-3-en-1-yl acetate & 1004 & 1004 & $\mathrm{~A}$ & $\mathrm{t}$ & 0.1 & & & & & & 1,2 \\
\hline (E)-Hex-2-en-1-yl acetate & 1007 & 1010 & A & & $\mathrm{t}$ & & & & & & 1,2 \\
\hline$\delta$-3-Carene* & 1008 & 1008 & M & 2.0 & 0.9 & 6.8 & 4.1 & 6.6 & 2.2 & 8.1 & 1,2 \\
\hline$\alpha$-Terpinene* & 1016 & 1014 & M & $\mathrm{t}$ & 0.1 & 0.2 & 0.1 & 0.1 & $\mathrm{t}$ & 0.4 & 1,2 \\
\hline p-Cymene* & 1020 & 1020 & M & & $\mathrm{t}$ & 0.1 & & $\mathrm{t}$ & $\mathrm{t}$ & & 1,2 \\
\hline$o$-Cymene & 1022 & 1022 & M & 0.1 & 0.1 & & & 0.8 & 0.2 & 0.4 & 1,2 \\
\hline Limonene* & 1026 & 1024 & M & 0.9 & 0.6 & 0.9 & 0.7 & 2.9 & 0.6 & 1.5 & $1,2,3$ \\
\hline$\beta$-Phellandrene*• & 1027 & 1025 & M & 0.1 & 0.5 & 1.9 & 1.5 & 1.0 & 0.3 & 6.5 & 1,2 \\
\hline 1,8-Cineole & 1031 & 1026 & M & & $\mathrm{t}$ & & & & & & $1,2,3$ \\
\hline (Z)- $\beta$-Ocimene*• & 1036 & 1032 & M & 0.1 & $\mathrm{t}$ & $\mathrm{t}$ & & $\mathrm{t}$ & $\mathrm{t}$ & $\mathrm{t}$ & 1,2 \\
\hline$(E)-\beta$-Ocimene*• & 1043 & 1034 & M & $\mathrm{t}$ & 0.1 & 0.5 & 0.3 & 0.1 & $\mathrm{t}$ & 1.0 & 1,2 \\
\hline$\gamma$-Terpinene*• & 1056 & 1054 & M & $\mathrm{t}$ & 0.1 & 0.9 & 0.3 & $\mathrm{t}$ & 0.1 & 1.1 & 1,2 \\
\hline (E)-Oct-2-en-1-ol & 1063 & 1060 & A & & $\mathrm{t}$ & & & & & & 1,2 \\
\hline cis-Sabinene hydrate• & 1068 & 1065 & M & 0.1 & 0.2 & & & $\mathrm{t}$ & & & 1,2 \\
\hline cis-Linalool oxide (furanoid) & 1074 & 1067 & M & & & & & & 0.2 & & 1,2 \\
\hline$m$-Cymenene & 1079 & 1082 & M & $\mathrm{t}$ & $\mathrm{t}$ & & & & & & 1,2 \\
\hline
\end{tabular}


TABLE 1 (Continued)

\begin{tabular}{|c|c|c|c|c|c|c|c|c|c|c|c|}
\hline \multirow{2}{*}{ Compound $^{\mathrm{a}}$} & \multirow{2}{*}{$\mathrm{RI}^{\mathrm{b}}$} & \multirow{2}{*}{ Lit. $\mathrm{RI}^{\mathrm{c}}$} & \multirow{2}{*}{ Class $^{\mathrm{d}}$} & \multicolumn{2}{|c|}{ Miramar } & \multicolumn{2}{|c|}{ San Pedro } & \multicolumn{3}{|c|}{ Pavas } & \multirow{2}{*}{$\begin{array}{l}\text { Ident. } \\
\text { methode }^{\text {e }}\end{array}$} \\
\hline & & & & Leaf & Flower & Leaf & Branch & Leaf & Flower & Branch & \\
\hline p-Mentha-2,4(8)-diene & 1080 & 1085 & M & & & $\mathrm{t}$ & & 0.1 & & & 1,2 \\
\hline Terpinolene* & 1084 & 1086 & M & $\mathrm{t}$ & 0.1 & 1.0 & 0.6 & $\mathrm{t}$ & 0.2 & 1.5 & 1,2 \\
\hline p-Cymenene & 1089 & 1089 & M & $\mathrm{t}$ & 0.1 & $\mathrm{t}$ & 0.1 & & & 0.2 & 1,2 \\
\hline Linalool* & 1094 & 1095 & M & 0.3 & 0.1 & $\mathrm{t}$ & $\mathrm{t}$ & 0.1 & 0.1 & & $1,2,3$ \\
\hline trans-Sabinene hydrate• & 1099 & 1098 & M & 0.1 & & $\mathrm{t}$ & & 0.1 & & & 1,2 \\
\hline Undecane & 1100 & 1100 & $\mathrm{~A}$ & & & & & & & $\mathrm{t}$ & 1,2 \\
\hline Nonanal & 1101 & 1100 & $\mathrm{~A}$ & $\mathrm{t}$ & 0.2 & & $\mathrm{t}$ & $\mathrm{t}$ & $\mathrm{t}$ & $\mathrm{t}$ & 1,2 \\
\hline Perillene & 1104 & 1102 & Misc & & $\mathrm{t}$ & & 0.1 & $\mathrm{t}$ & $\mathrm{t}$ & 1.0 & 1,2 \\
\hline$\alpha$-Fenchocamphorone & 1109 & 1104 & M & $\mathrm{t}$ & & & & & & & 1,2 \\
\hline (Z)-2-Isopropyl-5-methyl-hex-2-enal & 1112 & 1112 & $\mathrm{~A}$ & & $\mathrm{t}$ & & & & & & 1,2 \\
\hline trans-Thujone & 1113 & 1112 & M & $\mathrm{t}$ & & & & & & & 1,2 \\
\hline Dehydro-sabina ketone & 1116 & 1117 & M & 0.1 & & & & & & & 1,2 \\
\hline cis-p-Menth-2-en-1-ol*• & 1122 & 1118 & M & & 0.1 & 0.1 & $\mathrm{t}$ & 0.2 & $\mathrm{t}$ & 0.1 & 1,2 \\
\hline$\alpha$-Campholenal & 1123 & 1122 & M & 0.1 & & & & & & & 1,2 \\
\hline$(E, E)$-2,6-Dimethyl-1,3,5,7-octatetraene & 1128 & $1130^{\mathrm{h}}$ & M & & & $\mathrm{t}$ & & & & $\mathrm{t}$ & 1,2 \\
\hline cis-Limonene oxide & 1131 & 1132 & M & 0.1 & & & & $\mathrm{t}$ & 0.1 & & 1,2 \\
\hline cis-p-Mentha-2,8-dien-1-ol & 1133 & 1133 & M & & & & $\mathrm{t}$ & & & & 1,2 \\
\hline trans-Pinocarveol & 1135 & 1135 & M & $\mathrm{t}$ & 0.1 & & & & & & 1,2 \\
\hline trans-p-Menth-2-en-1-ol & 1136 & 1136 & M & & & $\mathrm{t}$ & & 0.1 & & $\mathrm{t}$ & 1,2 \\
\hline trans-Sabinol & 1136 & 1137 & M & $\mathrm{t}$ & & & & & & & 1,2 \\
\hline cis-Pinene hydrate & 1139 & 1139 & M & & & & & & $\mathrm{t}$ & & 1,2 \\
\hline (E)-Tagetone & 1139 & 1139 & M & 0.3 & & & & & & & 1,2 \\
\hline trans-Verbenol & 1144 & 1140 & M & & $\mathrm{t}$ & & & & 0.1 & & 1,2 \\
\hline p-Meth-3-en-8-ol & 1148 & 1145 & M & 0.2 & & & & & & & 1,2 \\
\hline Eucarvone & 1148 & 1146 & M & 0.2 & & & & & & & 1,2 \\
\hline neo-iso-Thujan-3-ol & 1149 & 1147 & M & & & & $\mathrm{t}$ & & & & 1,2 \\
\hline Nerol oxide & 1150 & 1154 & M & & & $\mathrm{t}$ & & $\mathrm{t}$ & & & 1,2 \\
\hline neo-Thujan-3-ol & 1153 & 1149 & M & $\mathrm{t}$ & & & & & & & 1,2 \\
\hline (E)-Non-2-enal & 1157 & 1157 & $\mathrm{~A}$ & & $\mathrm{t}$ & & $\mathrm{t}$ & & & & 1,2 \\
\hline Benzyl acetate & 1158 & 1157 & $\mathrm{~B}$ & 0.1 & & & & & & & 1,2 \\
\hline trans-Pinocamphone & 1160 & 1158 & M & & & & & & 0.1 & & 1,2 \\
\hline$\delta$-Terpineol & 1161 & 1162 & M & & 0.2 & & & & & & 1,2 \\
\hline (2Z)-Non-2-en-1-ol & 1163 & 1162 & $\mathrm{~A}$ & & & & & & 0.1 & & 1,2 \\
\hline 1,3-Dimetoxibenzene & 1165 & 1165 & $\mathrm{~B}$ & & 0.1 & & & & & & 1,2 \\
\hline Borneol & 1165 & 1165 & M & 0.1 & & $\mathrm{t}$ & & & & & 1,2 \\
\hline$p$-Mentha-1,5-dien-8-ol & 1167 & 1166 & M & & $\mathrm{t}$ & & $\mathrm{t}$ & $\mathrm{t}$ & $\mathrm{t}$ & & 1,2 \\
\hline Santolinyl acetate & 1173 & 1171 & M & & & & & & & $\mathrm{t}$ & 1.2 \\
\hline cis-Pinocamphone & 1175 & 1172 & M & 0.1 & 0.1 & & & & & & 1,2 \\
\hline Terpinen-4-ol*• & 1177 & 1174 & M & 0.7 & 0.1 & 0.5 & 0.3 & 0.7 & 0.1 & 0.8 & $1,2,3$ \\
\hline p-Methyl acetophenone & 1182 & 1179 & $\mathrm{~B}$ & $\mathrm{t}$ & & & & & & & 1,2 \\
\hline p-Cymen-8-ol & 1184 & 1179 & M & 0.4 & & $\mathrm{t}$ & & 0.7 & 0.1 & & 1,2 \\
\hline Cryptone• & 1186 & 1183 & M & & 0.1 & $\mathrm{t}$ & $\mathrm{t}$ & 0.1 & & & 1,2 \\
\hline Dill ether & 1188 & 1184 & M & & & & & & 0.2 & & 1,2 \\
\hline$\alpha$-Terpineol & 1190 & $1192^{\mathrm{i}}$ & M & 0.5 & & & $\mathrm{t}$ & 0.1 & 0.1 & 0.2 & 1,2 \\
\hline Methyl salicylate & 1191 & 1190 & $\mathrm{~B}$ & & & $\mathrm{t}$ & $\mathrm{t}$ & & & $\mathrm{t}$ & 1,2 \\
\hline Dihydrocarveol & 1192 & 1192 & M & $\mathrm{t}$ & & & & & & & 1,2 \\
\hline Myrtenol & 1196 & 1194 & M & & 0.1 & & & & & & 1,2 \\
\hline cis-Piperitol & 1198 & 1195 & M & & & $\mathrm{t}$ & & & & $\mathrm{t}$ & 1,2 \\
\hline Safranal & 1199 & 1197 & M & $\mathrm{t}$ & & & & & & & 1,2 \\
\hline
\end{tabular}


TABLE 1 (Continued)

\begin{tabular}{|c|c|c|c|c|c|c|c|c|c|c|c|}
\hline \multirow{2}{*}{ Compound $^{\mathrm{a}}$} & \multirow{2}{*}{$\mathrm{RI}^{\mathrm{b}}$} & \multirow{2}{*}{ Lit. RI ${ }^{\mathrm{c}}$} & \multirow{2}{*}{ Class $^{\mathrm{d}}$} & \multicolumn{2}{|c|}{ Miramar } & \multicolumn{2}{|c|}{ San Pedro } & \multicolumn{3}{|c|}{ Pavas } & \multirow{2}{*}{$\begin{array}{l}\text { Ident. } \\
\text { methode }^{\text {e }}\end{array}$} \\
\hline & & & & Leaf & Flower & Leaf & Branch & Leaf & Flower & Branch & \\
\hline Dodecane & 1200 & 1200 & A & & & & & & 0.1 & & $1,2,3$ \\
\hline Verbenone & 1206 & 1204 & M & 0.3 & 0.1 & & & & & & 1,2 \\
\hline trans-Piperitol & 1207 & 1207 & M & & & $\mathrm{t}$ & & $\mathrm{t}$ & & & 1,2 \\
\hline$(E, E)$-Nona-2,4-dienal & 1209 & 1210 & A & $\mathrm{t}$ & & & & & & & 1,2 \\
\hline 4-Methylene-isophorone & 1216 & 1216 & M & $\mathrm{t}$ & & & & 0.1 & & & 1,2 \\
\hline$\beta$-Cyclocitral & 1217 & 1217 & M & & 0.1 & & & & & & 1,2 \\
\hline cis-Sabinene-hydrate acetate & 1218 & 1219 & M & & & $\mathrm{t}$ & & & & $\mathrm{t}$ & 1,2 \\
\hline Citronellol & 1218 & 1219 & M & & & $\mathrm{t}$ & & & & & 1,2 \\
\hline (Z)-Ocimenone & 1228 & 1226 & M & & $\mathrm{t}$ & & & & & & 1,2 \\
\hline Nerol & 1230 & 1227 & M & & & $\mathrm{t}$ & & & & & 1,2 \\
\hline (3Z)-Hexenyl 3-methylbutanoate & 1232 & 1230 & $\mathrm{~A}$ & & & & & & & $\mathrm{t}$ & 1,2 \\
\hline (3Z)-Hexenyl 2-methylbutanoate & 1232 & 1232 & $\mathrm{~A}$ & & & $\mathrm{t}$ & & & & & 1,2 \\
\hline Ascaridole & 1234 & 1234 & M & & & & & $\mathrm{t}$ & & & 1,2 \\
\hline Cumin aldehyde & 1240 & 1238 & M & & 0.1 & & & & & & 1,2 \\
\hline Carvone & 1241 & 1239 & M & 0.2 & & & & & & & 1,2 \\
\hline (2Z)-Hexenyl 3-methylbutanoate & 1242 & 1241 & $\mathrm{~A}$ & & & & & $\mathrm{t}$ & & & 1,2 \\
\hline (2E)-Hexenyl 3-methylbutanoate & 1243 & 1243 & $\mathrm{~A}$ & & & & & 0.1 & & $\mathrm{t}$ & 1,2 \\
\hline Car-3-en-2-one & 1245 & 1244 & M & 0.1 & 0.1 & $\mathrm{t}$ & & 0.1 & & & 1,2 \\
\hline Piperitone & 1249 & 1249 & M & 0.1 & $\mathrm{t}$ & & & & & & 1,2 \\
\hline Geraniol & 1250 & 1249 & M & & $\mathrm{t}$ & & $\mathrm{t}$ & & & & 1,2 \\
\hline trans-Ascaridol glicol & 1267 & 1266 & M & & & & & 0.1 & & & 1,2 \\
\hline$p$-Menth-1-en-7-al & 1285 & 1273 & M & & $\mathrm{t}$ & & & & & $\mathrm{t}$ & 1,2 \\
\hline$p$-Ethyl acetophenone & 1285 & 1279 & $\mathrm{~B}$ & & & & $\mathrm{t}$ & & & & 1,2 \\
\hline Bornyl acetate & 1286 & 1287 & M & 0.1 & $\mathrm{t}$ & & & & & & 1,2 \\
\hline Thymol* & 1288 & 1289 & M & 0.3 & & & & & & & 1,2 \\
\hline$\gamma$-Terpinen-7-al & 1289 & 1291 & M & & & & $\mathrm{t}$ & & & & 1,2 \\
\hline Undecan-2-one & 1295 & 1293 & A & & $\mathrm{t}$ & & & & & & 1,2 \\
\hline Tridecane & 1300 & 1300 & $\mathrm{~A}$ & & 0.1 & & & & $\mathrm{t}$ & & $1,2,3$ \\
\hline Undecanal & 1308 & 1305 & A & & 0.1 & & & & 0.2 & & 1,2 \\
\hline Dihydrocarveol acetate & 1309 & 1306 & M & & & & & & $\mathrm{t}$ & & 1,2 \\
\hline Isoverbanol acetate & 1310 & 1308 & M & & & & & 0.5 & & & 1,2 \\
\hline$(E, E)$-Deca-2,4-dienal & 1314 & 1315 & $\mathrm{~A}$ & & $\mathrm{t}$ & & 0.1 & & & & 1,2 \\
\hline$\delta$-Terpinyl acetate & 1319 & 1316 & M & $\mathrm{t}$ & & & & & & & 1,2 \\
\hline (3Z)-Hexenyl tiglate & 1320 & 1319 & A & & & & & $\mathrm{t}$ & & & 1,2 \\
\hline Silphiperfol-5-ene & 1323 & 1326 & $\mathrm{~S}$ & & 0.1 & & & & & & 1,2 \\
\hline cis-Piperitol acetate & 1331 & 1332 & M & & 0.2 & & $\mathrm{t}$ & & & & 1,2 \\
\hline$\delta$-Elemene* & 1334 & 1335 & $\mathrm{~S}$ & & 0.2 & 0.3 & 0.2 & 0.1 & 0.2 & 0.1 & 1,2 \\
\hline 7-epi-Silphiperfol-5-ene & 1344 & 1345 & $\mathrm{~S}$ & & & $\mathrm{t}$ & $\mathrm{t}$ & & 0.1 & & 1,2 \\
\hline$\alpha$-Cubebene & 1345 & 1345 & $\mathrm{~S}$ & 0.4 & 0.3 & 0.5 & 0.7 & 0.2 & & 0.2 & 1,2 \\
\hline$\alpha$-Terpinyl acetate* & 1347 & 1346 & M & & & & & 0.2 & & & 1,2 \\
\hline$\alpha$-Longipinene & 1357 & 1350 & $\mathrm{~S}$ & $\mathrm{t}$ & & & & & 0.1 & & 1,2 \\
\hline Cyclosativene & 1366 & 1369 & $\mathrm{~S}$ & 0.5 & $\mathrm{t}$ & & $\mathrm{t}$ & & & & 1,2 \\
\hline$\alpha$-Ylangene & 1369 & 1373 & S & & 0.4 & 0.3 & 0.5 & 0.4 & 0.3 & 0.3 & 1,2 \\
\hline$\alpha$-Copaene*• & 1373 & 1374 & $\mathrm{~S}$ & 1.3 & 0.7 & 1.1 & 1.5 & 0.6 & 0.8 & 1.0 & 1,2 \\
\hline Silphiperfol-6-ene & 1377 & 1377 & $\mathrm{~S}$ & & 0.2 & & & & & & 1,2 \\
\hline$\beta$-Patchoulene & 1379 & 1379 & S & $\mathrm{t}$ & & & & & & & 1,2 \\
\hline$\beta$-Cubebene & 1384 & 1387 & $\mathrm{~S}$ & & 0.1 & $\mathrm{t}$ & & $\mathrm{t}$ & 0.3 & 0.1 & 1,2 \\
\hline$\beta$-Bourbonene & 1386 & 1387 & S & $\mathrm{t}$ & & & & 0.2 & & 0.1 & 1,2 \\
\hline$\beta$-Elemene*• & 1386 & 1389 & $\mathrm{~S}$ & 0.8 & 2.5 & 2.1 & 2.7 & 3.0 & 4.3 & 1.3 & 1,2 \\
\hline
\end{tabular}


TABLE 1 (Continued)

\begin{tabular}{|c|c|c|c|c|c|c|c|c|c|c|c|}
\hline \multirow{2}{*}{ Compound $^{\text {a }}$} & \multirow{2}{*}{$\mathrm{RI}^{\mathrm{b}}$} & \multirow{2}{*}{ Lit. $\mathrm{RI}^{\mathrm{c}}$} & \multirow{2}{*}{ Class $^{\mathrm{d}}$} & \multicolumn{2}{|c|}{ Miramar } & \multicolumn{2}{|c|}{ San Pedro } & \multicolumn{3}{|c|}{ Pavas } & \multirow{2}{*}{$\begin{array}{l}\text { Ident. } \\
\text { methode }^{\text {e }}\end{array}$} \\
\hline & & & & Leaf & Flower & Leaf & Branch & Leaf & Flower & Branch & \\
\hline Tetradecane & 1400 & 1400 & $\mathrm{~A}$ & & $\mathrm{t}$ & & & & $\mathrm{t}$ & & $1,2,3$ \\
\hline$\beta$-Longipinene & 1401 & 1400 & S & & 0.1 & $\mathrm{t}$ & 0.1 & & & & 1,2 \\
\hline Methyleugenol & 1403 & 1403 & PP & 0.2 & & $\mathrm{t}$ & & & & & 1,2 \\
\hline Longifolene & 1405 & 1407 & $\mathrm{~S}$ & & & $\mathrm{t}$ & $\mathrm{t}$ & & & & 1,2 \\
\hline$\alpha$-Gurjunene & 1409 & 1409 & S & & & & $\mathrm{t}$ & 0.2 & 0.1 & & 1,2 \\
\hline$\beta$-Caryophyllene*• & 1416 & 1417 & S & 3.5 & 9.5 & 9.1 & 12.4 & 7.9 & 17.1 & 10.1 & $1,2,3$ \\
\hline$\beta$-Copaene & 1428 & 1430 & S & 0.3 & 0.8 & $\mathrm{t}$ & 2.8 & 0.7 & $\mathrm{t}$ & 0.6 & 1,2 \\
\hline$\beta$-Gurjunene & 1429 & 1431 & S & & & & & & 0.7 & & 1,2 \\
\hline$\alpha$-trans-Bergamotene & 1432 & 1432 & S & $\mathrm{t}$ & 0.1 & & & & & 0.1 & 1,2 \\
\hline$\gamma$-Elemene & 1432 & 1434 & S & & & 1.9 & & $\mathrm{t}$ & & & 1,2 \\
\hline$\alpha$-Guaiene & 1434 & 1437 & S & & & 0.1 & & 0.1 & 0.1 & & 1,2 \\
\hline Aromadendrene & 1437 & 1439 & S & $\mathrm{t}$ & 0.1 & 0.2 & 0.1 & 0.3 & 0.1 & 0.2 & 1,2 \\
\hline$(Z)-\beta$-Farnesene & 1438 & 1440 & S & 0.1 & 0.3 & & & & & & 1,2 \\
\hline 6,9-Guaiadiene & 1442 & 1442 & S & & & 0.1 & $\mathrm{t}$ & $\mathrm{t}$ & $\mathrm{t}$ & & 1,2 \\
\hline cis-Muurola-3,5-diene & 1447 & 1448 & S & & $\mathrm{t}$ & & $\mathrm{t}$ & 0.2 & $\mathrm{t}$ & 0.1 & 1,2 \\
\hline trans-Muurola-3,5-diene & 1450 & 1451 & S & & & 0.2 & $\mathrm{t}$ & $\mathrm{t}$ & & 0.1 & 1,2 \\
\hline$\alpha$-Humulene*• & 1453 & 1452 & S & 0.5 & 2.2 & 1.0 & 1.3 & 1.1 & 1.8 & 1.4 & $1,2,3$ \\
\hline$(E)-\beta$-Farnesene & 1454 & 1454 & S & & $\mathrm{t}$ & & & & & & 1,2 \\
\hline Alloaromadendrene & 1458 & 1458 & S & 0.1 & & 0.1 & & & 0.1 & 0.1 & 1,2 \\
\hline cis-Cadina-1(6),4-diene & 1460 & 1461 & S & & & $\mathrm{t}$ & & & & 0.1 & 1,2 \\
\hline 9-epi- $\beta$-Caryophyllene & 1461 & 1464 & S & & & & & 0.1 & $\mathrm{t}$ & $\mathrm{t}$ & 1,2 \\
\hline cis-Muurola-4(14),5-diene & 1465 & 1465 & S & & 13.7 & & $\mathrm{t}$ & 0.1 & $\mathrm{t}$ & & 1,2 \\
\hline Dauca-5,8-diene & 1471 & 1471 & S & $\mathrm{t}$ & & & & & & & 1,2 \\
\hline trans-Cadina-1(6),4-diene & 1471 & 1475 & $\mathrm{~S}$ & $\mathrm{t}$ & & & & & & & 1,2 \\
\hline$\gamma$-Gurjunene & 1477 & 1475 & S & & & & & $\mathrm{t}$ & 0.6 & 0.3 & 1,2 \\
\hline$\gamma$-Muurolene*• & 1478 & 1478 & $\mathrm{~S}$ & & & & 2.1 & 1.6 & & 1.6 & 1,2 \\
\hline ar-Curcumene & 1471 & 1479 & S & 2.0 & 8.0 & & & & & & 1,2 \\
\hline$\gamma$-Curcumene & 1478 & 1481 & $\mathrm{~S}$ & 1.5 & & & & & & & 1,2 \\
\hline$\alpha$-Amorphene & 1483 & 1483 & S & 0.2 & & & 0.1 & & & & 1,2 \\
\hline Germacrene D* & 1486 & 1484 & $\mathrm{~S}$ & 0.5 & 4.3 & 19.1 & 14.7 & 6.3 & 9.9 & 15.6 & $1,2,3$ \\
\hline$\beta$-Selinene* & 1490 & 1489 & S & & 0.3 & 0.9 & 1.6 & 1.1 & 0.5 & & 1,2 \\
\hline cis- $\beta$-Guaiene & 1493 & 1492 & S & 0.6 & & & & & & & 1,2 \\
\hline trans-Muurola-4(14),5-diene & 1493 & 1493 & S & & $\mathrm{t}$ & 0.7 & & $\mathrm{t}$ & & & 1,2 \\
\hline Viridiflorene & 1496 & 1496 & $\mathrm{~S}$ & & & & 0.7 & & & 1.8 & 1,2 \\
\hline Bicyclogermacrene*• & 1498 & 1500 & S & & 8.3 & 4.4 & 3.6 & 2.5 & 5.9 & 4.9 & 1,2 \\
\hline$\alpha$-Muurolene* & 1497 & 1500 & $\mathrm{~S}$ & 0.5 & & & 1.0 & $\mathrm{t}$ & & 0.4 & 1,2 \\
\hline Epizonarene & 1502 & 1501 & $\mathrm{~S}$ & & & 0.7 & & & & & 1,2 \\
\hline trans- $\beta$-Guaiene & 1506 & 1502 & $\mathrm{~S}$ & & 0.5 & & & & & & 1,2 \\
\hline$\beta$-Bisabolene & 1505 & 1505 & $\mathrm{~S}$ & 0.4 & 1.4 & & & & & & 1,2 \\
\hline Germacrene A & 1507 & 1508 & S & & & 0.5 & 0.4 & 0.3 & & & 1,2 \\
\hline$\delta$-Amorphene & 1510 & 1511 & $\mathrm{~S}$ & 0.1 & 0.4 & & 0.4 & & 0.5 & 0.3 & 1,2 \\
\hline$\gamma$-Cadinene* & 1513 & 1513 & $\mathrm{~S}$ & 0.3 & 2.5 & 0.6 & 0.8 & 0.5 & 0.3 & 0.5 & 1,2 \\
\hline Cubebol & 1515 & 1514 & $\mathrm{~S}$ & 2.5 & 0.3 & 0.1 & $\mathrm{t}$ & & $\mathrm{t}$ & & 1,2 \\
\hline$\beta$-Sesquiphellandrene & 1518 & 1521 & $\mathrm{~S}$ & & 0.5 & & & & & & 1,2 \\
\hline$\delta$-Cadinene* & 1519 & 1522 & $\mathrm{~S}$ & 0.8 & & 3.4 & 4.3 & 1.4 & 1.4 & 3.5 & 1,2 \\
\hline cis-Calamenene & 1526 & 1528 & $\mathrm{~S}$ & 0.3 & & & 0.2 & $\mathrm{t}$ & & & 1,2 \\
\hline Zonarene & 1527 & 1528 & S & & & 0.1 & & & & 0.1 & 1,2 \\
\hline (Z)-Nerolidol & 1533 & 1531 & $\mathrm{~S}$ & 0.1 & & & & & & & 1,2 \\
\hline trans-Cadina-1,4-diene & 1531 & 1533 & $\mathrm{~S}$ & 0.2 & 0.5 & 0.2 & 0.2 & 0.2 & & & 1,2 \\
\hline
\end{tabular}


TABLE 1 (Continued)

\begin{tabular}{|c|c|c|c|c|c|c|c|c|c|c|c|}
\hline \multirow{2}{*}{ Compound $^{\mathrm{a}}$} & \multirow{2}{*}{$\mathrm{RI}^{\mathrm{b}}$} & \multirow{2}{*}{ Lit. RI ${ }^{\mathrm{c}}$} & \multirow{2}{*}{ Class $^{\mathrm{d}}$} & \multicolumn{2}{|c|}{ Miramar } & \multicolumn{2}{|c|}{ San Pedro } & \multicolumn{3}{|c|}{ Pavas } & \multirow{2}{*}{$\begin{array}{l}\text { Ident. } \\
\text { methode }^{\text {e }}\end{array}$} \\
\hline & & & & Leaf & Flower & Leaf & Branch & Leaf & Flower & Branch & \\
\hline 10-epi-Cubebol & 1535 & 1533 & S & 0.1 & & & & & & 0.3 & 1,2 \\
\hline$\alpha$-Cadinene* & 1536 & 1536 & S & & 0.2 & 0.4 & 0.6 & 0.2 & 0.1 & & 1,2 \\
\hline cis-Sesquisabinene hydrate & 1537 & 1542 & S & & & & & $\mathrm{t}$ & & & 1,2 \\
\hline$\alpha$-Calacorene & 1538 & 1544 & S & 0.7 & 0.5 & & & $\mathrm{t}$ & 0.1 & 0.2 & 1,2 \\
\hline Selina-3,7(11)-diene & 1541 & 1545 & S & & & 0.3 & 0.6 & & & & 1,2 \\
\hline Hedycaryol & 1546 & 1546 & S & & & & & 0.3 & & & 1,2 \\
\hline Elemol & 1547 & 1548 & S & & & & & & 0.2 & & 1,2 \\
\hline trans-Dauca-4(11),7-diene & 1552 & 1557 & S & & & & $\mathrm{t}$ & & & & 1,2 \\
\hline Silphiperfol-5-en-3-ol A & 1554 & 1557 & S & 0.1 & & & & & & & 1,2 \\
\hline Germacrene B* & 1557 & 1559 & S & 0.2 & 4.3 & 16.0 & 18.7 & 1.4 & 1.8 & 1.4 & 1,2 \\
\hline$\beta$-Calacorene & 1560 & 1564 & S & 1.3 & & & & & 0.2 & 0.4 & 1,2 \\
\hline (E)-Nerolidol & 1567 & 1561 & S & 0.2 & & & 0.1 & & & & $1,2,3$ \\
\hline Palustrol & 1569 & 1567 & S & 0.3 & 0.1 & 0.1 & 0.2 & 0.4 & 0.4 & 0.4 & 1,2 \\
\hline Dendrolasin & 1570 & 1570 & Misc & & 0.1 & & 0.1 & & & & 1,2 \\
\hline (Z)-Dihydroapofarnesol & 1571 & 1571 & $\mathrm{~S}$ & & $\mathrm{t}$ & & & & & 0.1 & 1,2 \\
\hline Spathulenol & 1577 & 1577 & S & 8.3 & 4.3 & 0.1 & 0.2 & 3.0 & 4.8 & 0.3 & 1,2 \\
\hline Caryophyllene oxide & 1583 & 1582 & S & 22.5 & 3.1 & 0.1 & 1.3 & 1.7 & 4.7 & 0.4 & 1,2 \\
\hline Thujopsan-2- $\alpha$-ol & 1585 & 1586 & S & & & & $\mathrm{t}$ & & & & 1,2 \\
\hline Globulol & 1590 & 1590 & S & & & 0.1 & 11.3 & 0.3 & 24.8 & 0.6 & 1,2 \\
\hline Salvial-4(14)-en-1-one & 1593 & 1594 & S & & $\mathrm{t}$ & & & & & & 1,2 \\
\hline Viridiflorol & 1594 & 1592 & S & 20.3 & 4.7 & 8.8 & & 21.0 & 0.3 & 11.5 & 1,2 \\
\hline Ledol & 1604 & 1602 & S & 0.5 & & 0.1 & 0.1 & 0.5 & & 0.3 & 1,2 \\
\hline 5-epi-7-epi- $\alpha$-Eudesmol & 1606 & 1607 & S & & & & $\mathrm{t}$ & 0.6 & & 0.5 & 1,2 \\
\hline Humulene epoxide II & 1608 & 1608 & S & 2.5 & 1.2 & & & & & & 1,2 \\
\hline 1,10-di-epi-Cubenol & 1616 & 1618 & S & & & $\mathrm{t}$ & & & & 0.3 & 1,2 \\
\hline Junenol & 1622 & 1618 & S & & & & $\mathrm{t}$ & & & 0.4 & 1,2 \\
\hline 1-epi-Cubenol & 1626 & 1627 & S & 2.1 & 0.7 & 0.1 & 0.2 & 0.4 & & 0.6 & 1,2 \\
\hline (E)-Sesquilavandulol & 1631 & 1631 & S & 0.3 & & & & & & & 1,2 \\
\hline cis-Cadin-4-en-7-ol & 1634 & 1635 & S & 1.6 & 3.5 & & & & & 0.5 & 1,2 \\
\hline epi- $\alpha$-Cadinol (=T-Cadinol) ${ }^{*}$ & 1641 & 1638 & S & 0.3 & 1.1 & 0.3 & 0.4 & 1.3 & 0.7 & $\mathrm{t}$ & 1,2 \\
\hline epi- $\alpha$-Muurolol (=T-Muurolol) & 1642 & 1640 & S & & 1.0 & 0.1 & 0.1 & 0.9 & 0.8 & 1.3 & 1,2 \\
\hline Cubenol & 1644 & 1645 & S & & 0.5 & & & & & & 1,2 \\
\hline$\alpha$-Muurolol (=Torreyol)* & 1645 & 1644 & $\mathrm{~S}$ & 0.5 & & $\mathrm{t}$ & $\mathrm{t}$ & & & 0.4 & 1,2 \\
\hline$\alpha$-Cadinol* & 1655 & 1652 & S & & 1.2 & 0.8 & 0.5 & 1.7 & 0.3 & 1.7 & 1,2 \\
\hline Selin-11-en-4- $\alpha$-ol & 1658 & 1658 & $\mathrm{~S}$ & & $\mathrm{t}$ & 0.1 & & 0.5 & 1.1 & 0.4 & 1,2 \\
\hline neo-Intermedeol & 1660 & 1658 & S & & & & 0.3 & & & & 1,2 \\
\hline cis-Calamenen-10-ol & 1663 & 1660 & S & & & & & 0.3 & & & 1,2 \\
\hline Intermedeol & 1665 & 1665 & S & & & & $\mathrm{t}$ & & & 0.1 & 1,2 \\
\hline trans-Calamenen-10-ol & 1667 & 1668 & $\mathrm{~S}$ & & & & & 0.3 & 0.2 & & 1,2 \\
\hline 14-Hydroxy-9-epi- $\beta$-caryophyllene & 1668 & 1668 & $\mathrm{~S}$ & 1.3 & & $\mathrm{t}$ & & & & & 1,2 \\
\hline Cadalene & 1672 & 1675 & S & $\mathrm{t}$ & 0.4 & & & & & & 1,2 \\
\hline Mustakone & 1672 & 1676 & $\mathrm{~S}$ & 0.5 & & & & & & & 1,2 \\
\hline Khusinol & 1679 & 1679 & S & & & & $\mathrm{t}$ & & & & 1,2 \\
\hline epi- $\alpha$-Bisabolol & 1683 & 1683 & $\mathrm{~S}$ & & 0.5 & & & & & & 1,2 \\
\hline Germacra-4(15),5,10(4)-trien-1- $\alpha$-ol & 1687 & 1685 & S & & & & & 0.4 & 0.4 & & 1,2 \\
\hline$\alpha$-Bisabolol & 1688 & 1685 & $\mathrm{~S}$ & & 0.5 & & & & & & 1,2 \\
\hline Eudesma-4(15),7-dien-1- $\beta$-ol & 1690 & 1687 & S & 0.6 & & & & & 0.1 & & 1,2 \\
\hline Eudesm-7(11)-en-4-ol & 1697 & 1700 & $\mathrm{~S}$ & & & $\mathrm{t}$ & 0.1 & & & & 1,2 \\
\hline Heptadecane & 1700 & 1700 & $\mathrm{~A}$ & & & & & & 0.2 & & $1,2,3$ \\
\hline
\end{tabular}


TABLE 1 (Continued)

\begin{tabular}{|c|c|c|c|c|c|c|c|c|c|c|c|}
\hline \multirow{2}{*}{ Compound $^{\text {a }}$} & \multirow{2}{*}{$\mathrm{RI}^{\mathrm{b}}$} & \multirow{2}{*}{ Lit. $\mathrm{RI}^{\mathrm{c}}$} & \multirow{2}{*}{ Class $^{d}$} & \multicolumn{2}{|c|}{ Miramar } & \multicolumn{2}{|c|}{ San Pedro } & \multicolumn{3}{|c|}{ Pavas } & \multirow{2}{*}{$\begin{array}{l}\text { Ident. } \\
\text { methode }^{\text {e }}\end{array}$} \\
\hline & & & & Leaf & Flower & Leaf & Branch & Leaf & Flower & Branch & \\
\hline Amorpha-4,9-dien -2-ol & 1699 & 1700 & $\mathrm{~S}$ & $\mathrm{t}$ & & & & & & & 1,2 \\
\hline 10-Nor-Calamenen-10-one & 1701 & 1702 & B & $\mathrm{t}$ & & & & & & & 1,2 \\
\hline Pentadecanal & 1724 & $1717^{\mathrm{j}}$ & A & & 0.3 & & 0.1 & & 0.3 & & 1,2 \\
\hline Isobicyclogermacrenal & 1730 & 1733 & S & $\mathrm{t}$ & & & & & & & 1,2 \\
\hline Mint sulfide & 1738 & 1740 & S & & 0.5 & & & 0.6 & 0.4 & 0.1 & 1,2 \\
\hline 2- $\alpha$-Hydroxy-amorpha-4,7(11)-diene & 1775 & 1775 & S & $\mathrm{t}$ & & & 0.3 & & & & 1,2 \\
\hline Hexadecanal & 1836 & 1836 & $\mathrm{~A}$ & & & & & & & 0.1 & 1,2 \\
\hline 6,10,14-Trimethyl-2-pentadecanone & 1848 & 1848 & IT & & 0.3 & & & & & & 1,2 \\
\hline Farnesyl acetone & 1886 & 1889 & IT & & & & $\mathrm{t}$ & & & & 1,2 \\
\hline Hexadecanoic acid & 1959 & 1959 & $\mathrm{~A}$ & & & & 0.3 & & & & $1,2,3$ \\
\hline Ethyl hexadecanoate & 1992 & 1993 & $\mathrm{~A}$ & & & & & & & 0.1 & 1,2 \\
\hline$(Z, E)$-Geranyl linalool & 1998 & 1997 & $\mathrm{D}$ & & & & & & & 0.2 & 1,2 \\
\hline (6E,10Z)-pseudo Phytol & 2016 & 2018 & $\mathrm{D}$ & & 0.2 & & & & & & 1,2 \\
\hline (Z)-Phytol & 2014 & 2014 & $\mathrm{D}$ & & 0.1 & & & & & 0.2 & 1,2 \\
\hline (E)-Phytol & 2106 & 2107 & $\mathrm{D}$ & & & & & $\mathrm{t}$ & & & 1,2 \\
\hline Linolenic acid & 2132 & 2129 & $\mathrm{~A}$ & & & & & & & 0.1 & 1,2 \\
\hline Docosane & 2200 & 2200 & $\mathrm{~A}$ & & $\mathrm{t}$ & & & & & & $1,2,3$ \\
\hline Tricosane & 2300 & 2300 & $\mathrm{~A}$ & & $\mathrm{t}$ & & & & & & $1,2,3$ \\
\hline Pentacosane & 2500 & 2500 & A & & $\mathrm{t}$ & & & & & & $1,2,3$ \\
\hline Hexacosane & 2600 & 2600 & $\mathrm{~A}$ & & $\mathrm{t}$ & & & & & & $1,2,3$ \\
\hline Heptacosane & 2700 & 2700 & $\mathrm{~A}$ & & $\mathrm{t}$ & & & & & & $1,2,3$ \\
\hline Octacosane & 2800 & 2800 & $\mathrm{~A}$ & & $\mathrm{t}$ & & & & & & $1,2,3$ \\
\hline Nonacosane & 2900 & 2900 & $\mathrm{~A}$ & & $\mathrm{t}$ & & & & & & $1,2,3$ \\
\hline Triacontane & 3000 & 3000 & A & & $\mathrm{t}$ & & & & & & $1,2,3$ \\
\hline Untriacontane & 3100 & 3100 & $\mathrm{~A}$ & & $\mathrm{t}$ & & & & & & $1,2,3$ \\
\hline Total & & & & 97.3 & 95.6 & 96.0 & 98.6 & 93.1 & 93.4 & 96.8 & \\
\hline Compounds & & & & 121 & 129 & 92 & 93 & 102 & 84 & 90 & \\
\hline
\end{tabular}

${ }^{\mathrm{a} C}$ Compounds listed in order of elution from $5 \%$ phenyl $95 \%$ dimethylpolysiloxane column. ${ }^{\mathrm{b}} \mathrm{RI}=$ Retention index relative to $\mathrm{C}_{8}-\mathrm{C}_{32} n$-alkanes on the $5 \%$ phenyl $/ 95 \%$ dimethylpolysiloxane column. ${ }^{\mathrm{c}} \mathrm{Lit}$. $\mathrm{RI}=\left(\right.$ Adams, 2007 ). ${ }^{\mathrm{d}}$ Compound class: A, aliphatics; B, benzenoids; D, diterpenoids; IT, irregular terpenoids; M, monoterpenoids; Misc, miscellaneous; PP, phenylpropanoids; S, sesquiterpenoids. ${ }^{~}$ Method: $1=$ Retention index on $5 \%$ phenyl $/ 95 \%$ dimethylpolysiloxane. $2=$ MS spectra. 3 = Standard. ${ }^{\mathrm{f}}\left(\right.$ Jordan, Margaria, Shaw, \& Goodner, 2002). ${ }^{\mathrm{g}}$ (Radulovic, Dordevic, \& Palic, 2010). ${ }^{\mathrm{h}}$ (Ali et al., 2008). ${ }^{i}$ (Zoghbi et al., 1998). j(Flamini et al., 2003).

$\mathrm{t}$ : traces $(<0.05 \%)$. Blank space $=$ not detected. Asterisk $*=$ compounds reported previously in Albuquerque et al. (2004) and Sobrinho et al. (2016). Bullet $=$ compounds reported in Rojas et al. (2008).

the percentages of the various classes of constituents of the essential oils are indicated. In total, 268 compounds were identified by means of GC-FID and GC-MS techniques, which represented 95.6 to $98.6 \%$ of the total essential oil compositions.

Samples of the species were gathered in three different Costa Rican locations, showing qualitative similarities, but some major quantitative differences. The leaf essential oil obtained from Miramar was dominated by oxygenated sesquiterpenes (64.6\%), with caryophyllene oxide (22.5\%) (Fig. 1), viridiflorol (20.3\%) and spathulenol $(8.3 \%)$ as principal components, accompanied by lesser amounts in the 1.5$3.9 \%$ range, namely $\alpha$-pinene, $\beta$-caryophyllene, cubebol, humulene epoxide II, 1-epi-cubenol, $\delta$-3-carene, ar-curcumene, cis-cadin-4-en-7-ol, $\beta$-pinene, and $\gamma$-curcumene. The main constituents of the leaf essential oil from San Pedro 


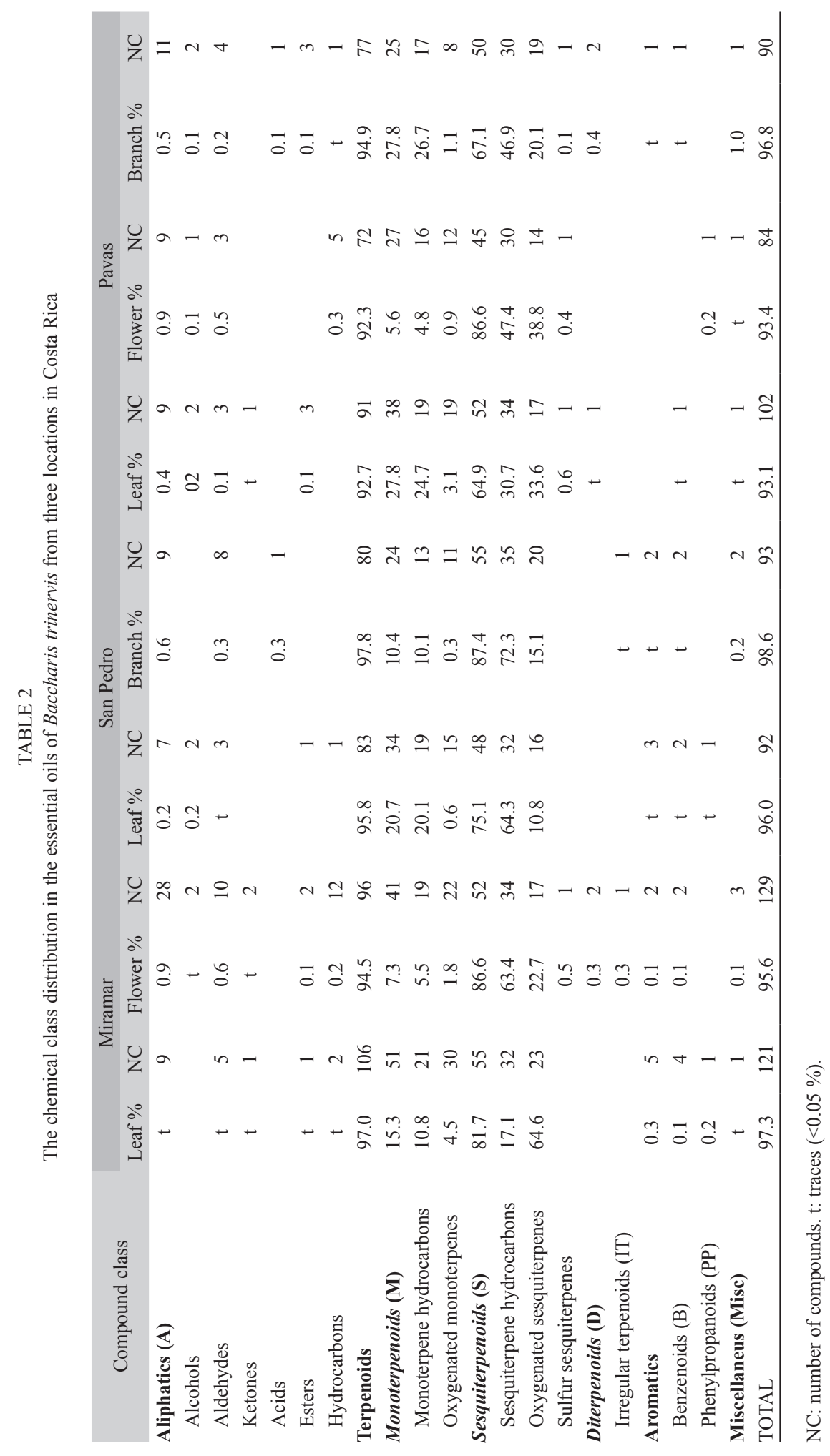




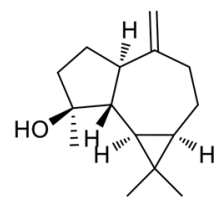

A

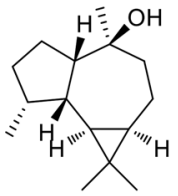

B

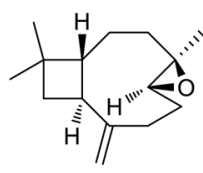

C

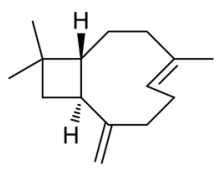

D

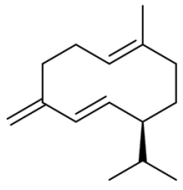

E

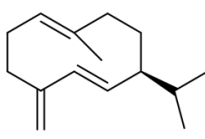

$\mathbf{F}$

Fig. 1. Chemical structures of some principal sesquiterpenic constituents of Baccharis trinervis essential oil from Costa Rica: a) Spathulenol, b) viridiflorol, c) caryophyllene oxide, d) $\beta$-caryophyllene, e) germacrene D, and f) germacrene B.

were germacrene D (19.1\%), germacrene B (16.0\%), $\beta$-caryophyllene (9.1\%), viridiflorol $(8.8 \%)$, and $\delta$-3-carene $(6.8 \%)$. Other significant constituents in the 1.3-3.4\% range were $\delta$-cadinene, $\alpha$-pinene, $\beta$-elemene, $\gamma$-elemene, $\beta$-phellandrene, $\alpha$-thujene, and $\beta$-pinene. The main components of Pavas leaf essential oil were viridiflorol $(21.0 \%), \beta$-caryophyllene (7.9 \%), $\delta$-3-carene $(6.6 \%)$, germacrene D (6.3\%), and $\alpha$-pinene $(5.9 \%)$. These compounds were accompanied by other significant constituents in the 1.4-3.0\% range, namely $\beta$-elemene, spathulenol, limonene, $\beta$-pinene, bicyclogermacrene, $\alpha$-cadinol, caryophyllene oxide, $\alpha$-thujene, sabinene, $\gamma$-muurolene, $\delta$-cadinene, and germacrene B.

The composition of the flower essential oil obtained from samples collected in the localities of Miramar and Pavas were dominated by sesquiterpenoids $(86.6 \%)$ with cis-muurola-4(14),5-diene (13.7\%), $\beta$-caryophyllene $(9.5 \%)$, bicyclogermacrene $(8.3 \%)$, and arcurcumene $(8.0 \%)$ as major constituents, accompanied by lesser amounts of several compounds in the 2.2-4.7\% range, namely viridiflorol, germacrene $\mathrm{D}$, germacrene $\mathrm{B}$, spathulenol, cis-cadin-4-en-7-ol, $\gamma$-cadinene, $\beta$-elemene, and humulene or globulol $(24.8 \%), \beta$-caryophyllene $(17.1 \%)$, germacrene D $(9.9 \%)$, bicyclogermacrene (5.9\%), spathulenol $(4.8 \%)$, caryophyllene oxide $(4.7 \%)$, and $\beta$-elemene $(4.3 \%)$. The branch essential oil from San Pedro sample also was constituted mainly by sesquiterpenoids $(87.4 \%)$ with germacrene B $(18.7 \%)$, germacrene D (14.7\%), $\beta$-caryophyllene (12.4\%) and globulol $(11.3 \%)$ as main constituents. There were also lesser amounts of several compounds in the 2.2-4.3\% range, namely $\delta$-cadinene, bicyclogermacrene, $\beta$-copaene, $\beta$-elemene, and $\delta$-3-carene. The branch essential oil from Pavas sample was constituted mainly by sesquiterpenoids $(67.1 \%)$ with more quantity of monoterpenoids $(27.8 \%)$ than the sample from San Pedro $(10.4 \%)$. The main compounds were germacrene D (15.6\%), viridiflorol (11.5\%), $\beta$-caryophyllene $(10.1 \%), \delta$-3-carene $(8.1 \%)$, $\beta$-phellandrene $(6.5 \%)$, bicyclogermacrene $(4.9 \%)$, and $\delta$-cadinene $(3.5 \%)$.

As can be seen from table 1 and table 2, the major constituents of the leaf essential oil from the sample collected in Miramar were oxygenated sesquiterpenes $(64.6 \%)$, whereas sesquiterpene hydrocarbons $(64.3 \%)$ were the major components of the San Pedro sample. However, in the sample from Pavas, the two cited classes of metabolites were almost equally distributed (30.7 and $33.6 \%$, respectively).

\section{DISCUSSION}

The essential oil of fresh aerial parts of Baccharis trinervis, collected on Merouca mountain region of Ceará state, Northeastern Brazil (Albuquerque et al., 2004; Sobrinho et al., 2016), revealed that the major constituents were terpenoids with the presence of $\beta$-phellandrene (18.4-27.8\%), sabinene (10.9$14.2 \%), \quad \alpha$-thujene (6.6-10.5 \%), (Z)- $\beta$ ocimene (2.3-8.1 \%), $\alpha$-pinene (5.5-8.7 \%), and $(E)-\beta$-ocimene (1.9-6.3\%), including two non-terpenoid $\mathrm{C}-10$ ene-diyne esters: methyl (Z)-dec-2-en-4,6-diynoate (0.8-14.6\%) and methyl $(E)$-dec-2-en-4,6-diynoate (10.7-14.7 $\%$ ) not found in other oils of Baccharis species studied up to day. Rojas et al. (2008) examined 
the essential oil from leaves collected on Santa Rosa, La Hechicera, in Mérida State (Venezuela). In this study, the authors reported that the major constituents were germacrene D (20.1 $\%)$, limonene $(15.0 \%), \delta$-cadinene $(5.2 \%)$, $\beta$-caryophyllene (4.8\%), $\alpha$-pinene (4.5\%), and bicyclogermacrene $(4.0 \%)$, and this oil can be distinguished from the Brazilian ones in the fact that the main C-10 ene-diyne esters were absent. Our experimental data support those obtained by Rojas et al. (2008) because the results of the essential oil samples from the three different locations in Costa Rica do not contain those characteristic and very specific $\mathrm{C}-10$ ene-diyne esters found in the Brazilian botanical material. As an additional support, the phytochemical study realized by Bohlmann and Zdero (1970) of fresh aerial parts of $B$. trinervis, cultured from seeds -of not specified origin- at Botanical Garden of the University of Berkeley, verified the presence of matricaria ester and three $\mathrm{C}-17$ ene-diyne-diene esters but not C-10 ene-diyne esters (see also, Bohlmann, Burkhardt, \& Zdero, 1973). Nonetheless, in a later phytochemical study of nine species of Baccharis collected in Brazil, the aerial parts of $B$. trinervis afforded, besides several terpenoids, one compound which has the trivial name lachnophyllum ester (probably, its $Z$ isomer) corresponding to methyl dec-2-en-4,6-diynoate (Bohlmann et al., 1981). This was the first diacetylenic compound with established chemical constitution isolated from an essential oil (Sørensen, 1977). This significant variation in the qualitative composition of the essential oils of the same species collected in Venezuela and Costa Rica compared with the Brazilian samples could be caused by the habitat and environment factors as well as the genotype of the plant, but real sources of variability, according to Németh-Zámboriné (2016), are 'hard to determine'. Furthermore, it is known that Baccharis is a diverse and complex genus of the Asteraceae because some of the species present a high degree of morphological and chemical variability. One of the distinguishing factors of the oils from this plant growing wild in Costa Rica is the presence of an array of terpenoids with diverse carbon skeletons that could arise through various biosynthetic patterns. Especially important in number are the cadinane class and the guaiane family of sesquiterpenoids that are originated from the germacrane biosynthetic pathway. A distinctive character of the oils of $B$. trinervis studied from Costa Rica is the presence of the oxygenated sesquiterpenoids spathulenol, viridiflorol and globulol, in important amounts, accompanied by lesser quantities of several compounds of the same carbon skeleton, that are not found in the Venezuelan sample nor in the oils of Brazilian botanical material. These compounds also were found in B. articulata (Lam.) Pers. (Minteguiaga et al., 2015), B. caprariifolia DC. (Ferracini et al., 1995), B. crispa Spreng. (Simões-Pires et al., 2005), B. dracunculifolia DC. (Frizzo et al., 2008; Fabiane, Ferronatto, dos Santos, \& Onofre, 2008), B. erioclada DC. (Ferracini et al., 1995), B. platipoda DC. (Ferracini et al., 1995; Quiroga, Ferracini, \& Marsaioli, 1996), B. semiserrata DC. (Vannini et al., 2012), B. tridentata Vahl (Ferracini et al., 1995; Quiroga et al., 1996), B. trimera (Less.) DC. (Silva et al., 2007; Oliveira et al., 2012), B. uncinella (Fabiane et al., 2008), and B. vincaefolia Baker (Ferracini et al., 1995). Spathulenol appear to be a widespread compound in the essential oils from plants of the genus Baccharis. Our findings corroborate the presence of 41 compounds previously reported (Albuquerque et al., 2004; Sobrinho et al., 2016; Rojas et al., 2008) indicated by asterisks and bullets in table 1, whereas 227 constituents are newly reported in the composition of oils from Baccharis trinervis.

The aerial parts of Baccharis trinervis growing wild in Costa Rica produce terpenoid-rich essential oils whose compositions were dominated by either globulol (0-24.8\%), caryophyllene oxide (0.1-22.5\%), viridiflorol (0-21.0\%), germacrene D (0.5-19.1\%), germacrene B (0.2-18.7\%), $\beta$-caryophyllene (3.5$17.1 \%)$, cis-muurola-4(14),5-diene (0-13.7\%), bicyclogermacrene (0-8.3\%), spathulenol (0.1$8.3 \%)$, ar-curcumene $(0-8.0 \%), \delta$-3-carene (0.9-6.8\%), or $\alpha$-pinene (0.3-5.9\%), according 
to the morphological part studied or the locality of collection of the sample. The composition of the essential oils of $B$. trinervis from central Costa Rica is qualitatively different to the composition of the oils from samples of the same species collected in the state of Ceará, Brazil, which contain two characteristic stereoisomeric lachnophyllum esters, compounds not detected in this study. The essential oils of plants from Costa Rica resemble the composition of the oil from Venezuelan origin, but differ from both Brazilian and Venezuelan essential oils by the presence of sesquiterpenoids of the guaiane carbon skeleton, some of them as prominent members: viridiflorol, globulol, and spathulenol.

\section{ACKNOWLEDGMENTS}

The authors are grateful to Escuela de Química and Vicerrectoría de Investigación (UCR) for financial support (Project No. 809B1-190) and to C. O. Morales (Biology School, UCR) for the species identification.

\section{RESUMEN}

Constituyentes de los aceites esenciales de Baccharis trinervis (Asteraceae) de Costa Rica. Baccharis (Asteraceae) es un género de plantas con flor que consta de 340 a 400 especies que habitan desde el sur de EE. UU. hasta Argentina y Chile, incluyendo América Central y varias islas del Caribe. Baccharis trinervis es un arbusto nativo de México, América Central y América del Sur. En Costa Rica, esta especie se conoce popularmente como alcotán y las hojas frescas se utilizan en forma de cataplasma para curar heridas y úlceras. El objetivo del presente estudio fue el de identificar los constituyentes químicos de los aceites esenciales obtenidos de diferentes partes morfológicas de B. trinervis en tres localidades de Costa Rica, obtenidos mediante el método de hidrodestilación. Se analizó la composición química de los aceites por cromatografía capilar de gases con detector de ionización de flama (GCFID) y cromatografía de gases acoplada a un detector de masas (GC-MS), utilizando los índices de retención en una columna tipo DB-5 y los patrones de fragmentación, lo cual permitió la identificación de 268 constituyentes. Los siete aceites están constituidos principalmente por terpenoides (92.3 a $97.8 \%$ ). Los compuestos mayoritarios de los aceites de las hojas se identificaron como óxido de cariofileno (0.1-22.5\%), viridiflorol (8.8-21.0\%), germacreno D (0.5-19.1\%), germacreno B (0.2-16.0\%), $\beta$-cariofileno
(3.5-9.1\%), espatulenol (0.1-8.3\%), $\delta$-3-careno (2.0$6.8 \%$ ), $\alpha$-pineno (2.5-5.9\%), biciclogermacreno (0-4.4\%), $\delta$-cadineno (0.8-3.4\%), $\beta$-elemeno (0.8-3.0\%), limoneno (0.9-2.9\%) y $\beta$-pineno (1.3-2.5\%). Los aceites de las flores contienen principalmente globulol (0-24\%), $\beta$-cariofileno (9.5-17.1\%), cis-muurola-4(14),5-dieno (t-13.7\%), germacreno D (4.3-9.9\%), biciclogermacreno (5.9-8.3\%), ar-curcumeno (0-8.0 \%), espatulenol (4.3-4.8\%), óxido de cariofileno (3.1-4.7\%), viridiflorol (0.3-4.7\%), $\beta$-elemeno (2.5-4.3\%), germacreno B (1.8-4.3\%), $\gamma$-cadineno $(0.3-$ $2.5 \%), \delta$-3-careno (0.9-2.2\%) y $\alpha$-humuleno (1.8-2.2\%). Los constituyentes mayoritarios del aceite de las ramitas fueron: germacreno B (1.4-18.7\%), germacreno D (14.7-15.6\%), $\beta$-cariofileno (10.1-12.4\%), viridiflorol (0-11.5\%), globulol (0.6-11.3\%), $\delta$-3-careno (4.1-8.1\%), $\beta$-felandreno (1.5-6.5\%), biciclogermacreno (3.6-4.9\%), $\delta$-cadineno (3.5-4.3\%), $\beta$-copaeno (0.6-2.8 \%), $\beta$-elemeno (1.3-2.7\%) y $\gamma$ - muuroleno (1.6-2.6\%). Los aceites estudiados presentan una composición compleja y se diferencian de los aceites obtenidos de la misma especie que crece en Brasil por la ausencia de los compuestos isoméricos diacetilénicos dec-2-en-4,6-diinoato de metilo ( $Z$ y $E)$. También se diferencian de los aceites de las plantas de Brasil y Venezuela por la presencia de sesquiterpenoides de la familia de los guayanos, en especial por cantidades apreciables de viridiflorol, globulol y espatulenol.

Palabras clave: Baccharis trinervis, Asteraceae, aceites esenciales, terpenoides, GC-MS, Costa Rica.

\section{REFERENCES}

Abad, M. J., Bermejo, P., Sánchez-Palomino, S., Chiriboga, X., \& Carrasco, L. (1999). Antiviral activity of some South American medicinal plants. Phytotherapy Research, 13, 142-146.

Abad, M. J., \& Bermejo, P. (2007). Baccharis (Compositae): a review update. ARKIVOC, Part (vii), 76-96.

Adams R. P. (2007). Identification of Essential Oil Components by Gas Chromatography / Mass Spectrometry $\left(4^{\text {th }}\right.$ ed). Carol Stream, Illinois: Allured Publishing Corporation.

Ali, N. A. A., Wurster, M., Arnold, N., Teichert, A., Schmidt, J., Lindequist, U., \& Wessjohann, L. (2008). Chemical composition and biological activities of essential oils from the oleogum resins of three endemic soqotraen Boswellia species. Records of Natural Products, 2(1), 6-12.

Albuquerque, M. R. J. R., Souza, E. B., Lins, M. U. D. S., Nogueira, N. A. P., Lemos, T. L. G., Silveira, E. R., \& Pessoa, O. D. L. (2004). Composition and antimicrobial activity of the essential oil from aerial parts of Baccharis trinervis (Lam.) Pers. ARKIVOC, Part (vi), 59-65. 
Bohlmann, F., \& Zdero, C. (1970). Polyacetylenverbindungen. 179. Über die Inhaltsstoffe von Baccharis trinervis Pers. Chemische Berichte, 103(8), 2327-2329.

Bohlmann, F., Burkhardt, T., \& Zdero, C. (1973). Naturally occurring acetylenes (pp. 215-217). London: Academic Press.

Bohlmann, F., Kramp, W., Grenz, M., Robinson, H., \& King, R. M. (1981). Diterpenes from Baccharis species. Phytochemistry, 20(8), 1907-1913.

Ferracini, V. L., Paraiba, L. C., Leitao Filho, H. F., da Silva, A. G., Nascimento, L. R., \& Marsaioli, A. J. (1995). Essential oils of seven Brazilian Baccharis species. Journal of Essential Oil Research, 7(4), 355-67.

Fabiane, K. C., Ferronatto, R., dos Santos, A. C., \& Onofre, S. B. (2008). Physicochemical characteristics of the essential oils of Baccharis dracunculifolia and Baccharis uncinella DC. (Asteraceae). Revista Brasileira de Farmacognosia, 18(2), 197-203.

Flamini, G., Cioni, P. L, Morelli, I., Ceccarini L., Andolfi, L., \& Macchia, M. (2003). Composition of the essential oil of Medicago marina L. from the coastal dunes of Tuscany, Italy. Flavour and Fragrance Journal, $18,460-462$.

Frizzo, C. D., Atti-Serafini, L., Laguna, S. E., Cassel, E., Lorenzo, D., \& Dellacassa, E. (2008). Essential oil variability in Baccharis uncinella DC and Baccharis dracunculifolia DC growing wild in southern Brazil, Bolivia and Uruguay. Flavour and Fragrance Journal, 23(2), 99-106.

Heiden, G., Andrade-Baumgratz, J. F., \& Esteves, R. L. (2012). Baccharis subgen. Molina (Asteraceae) no estado do Rio de Janeiro, Brasil. Rodriguésia, 63(3), 649-687.

Heras, B., Slowing, K., Benedí, J., Carretero, E., Ortega, T., Toledo, C., Bermejo, P., ..., \& Villar, A. (1998). Antiinflammatory and antioxidant activity of plants used in traditional medicine in Ecuador. Journal of Ethnopharmacology, 61, 161-166.

Herrera, J. C., Rosas-Romero, A. J., Crescente, O. E., Acosta, M., \& Pekerar, S. (1996). Analysis of 5-hydroxy-7-methoxyflavones by normal-phase high-performance liquid chromatography. Journal of Chromatography A, 740, 201-206.

House, P. R., Lagos-Witte, S., Ochoa, L., Torres, C., Mejía, T., \& Rivas, M. (1995). Plantas medicinales comunes de Honduras (pp. 50-51). Tegucigalpa, Honduras: UNAH, CIMN-H, CID/CIIR, GTZ.

Jordan, M. J., Margaria, C. C., Shaw, P. E., \& Goodner, K. L. (2002). Aroma active components in aqueous Kiwi fruit essence and Kiwi fruit puree by GC-MS and multidimensional GC/GC-O. Journal of Agricultural and Food Chemistry, 50, 5386-5390.
Kuroyanagi, M., Uchida, K., Ueno, A., Satake, M., \& Shimomura, K. (1993). Neo-clerodane type diterpenes from Baccharis trinervis. Phytochemistry, 34(5), 1377-1384.

León, J., \& Poveda, L. J. (2000). In P. E. Sánchez-Vindas (Ed.), Nombres comunes de las plantas en Costa Rica (pp. 48). San José, Costa Rica: Editorial Guayacán.

Minteguiaga, M., Umpiérrez, N., Fariña, L., Falcão, M. A., Xavier, V. B., Cassel, E., \& Dellacassa, E. (2015). Impact of gas chromatography and mass spectrometry combined with gas chromatography and olfactometry for the sex differentiation of Baccharis articulata by the analysis of volatile compounds. Journal of Separation Science, 38(17), 2931-3118.

Németh-Zámboriné, E. (2016). Natural variability of essential oil components. In K. H. C. Başir, \& G. Buchbauer (Eds.). Handbook of essential oils: Science, technology, and applications (pp. 87-125). Boca Raton, FL: CRC Press/Taylor \& Francis.

Núñez-Meléndez, E. (1978). Plantas medicinales de Costa Rica y su folklore. San José, Costa Rica: Editorial Universidad de Costa Rica.

Oliveira, R. N., Rehder, V. L., Oliveira, A. S. S., Júnior, I. M., de Carvalho, J. E., de Ruiz, A. L. T., Jeraldo, V. L. S., Linhares, A. X., \& Allegretti, S. M. (2012). Schistosoma mansoni: In vitro schistosomicidal activity of essential oil of Baccharis trimera (Less.) DC. Experimental Parasitology, 132, 135-143.

Pittier, H. (1978). Plantas usuales de Costa Rica (reprint of 2nd ed., 1957). San José, Costa Rica: Editorial Costa Rica.

Quiroga, C. L., Ferracini, V. L., \& Marsaioli, A. J. (1996). Three new oxigenated cadinanes from Baccharis Species. Phytochemistry, 42(4), 1097-1103.

Radulovic, N. S., Dordevic, N. D., \& Palic, R. (2010). Volatile of Pleurospermim austriacum (L.) Hoffm. (Apiaceae). Journal of Serbian Chemical Society, 75(12), 1-11.

Ramírez-Cárdenas, A., Isaza-Mejía, G. \& Pérez-Cárdenas, J. E. (2013). Especies vegetales investigadas por sus propiedades antimicrobianas, inmunomoduladoras e hipoglicemientes en el Departamento de Caldas (Colombia, Sudamérica). Biosalud, 12(1), 59-82.

Ramos Campos, F., Bressan, J., Godoy Jasinski, V. C., Zuccolotto, T., da Silva, L. E., \& Bonancio Cerqueira, L. (2016). Baccharis (Asteraceae): Chemical constituents and biological activities. Chemistry and Biodiversity, 13, 1-17.

Rojas, J., Velasco, J., Morales, A., Rojas, L., Díaz, T., Rondón, M., \& Carmona, J. (2008). Chemical composition and antibacterial activity of the essential oil of Baccharis trinervis (Lam.) Pers. (Asteraceae) 
collected in Venezuela. Natural Product Communications, 3(3), 369-372.

Sánchez-Palomino, S., Abad, M. J., Bedoya, L. M., García J., Gonzales, E., Chiriboga, X., Bermejo, P., \& Alcami, J. (2002). Screening of South American plants against human immunodeficiency virus: Preliminary fractionation of aqueous extract from Baccharis trinervis. Biological and Pharmaceutical Bulletin, $25,1147-1150$

Sharp, H., Bartholomew, B., Bright, C., Latif, Z., Sarker S. D., \& Nash, R. J. (2001). 6-Oxygenated flavones from Baccharis trinervis (Asteraceae). Biochemical Systematics and Ecology, 29, 105-107.

Silva, F. G., Oliveira, C. B. A., Pinto, J. E. B. P., Nascimento, V. E., Santos, S. C., Seraphin, J. C., \& Ferri, P. H. (2007). Seasonal variability in the essential oils of wild and cultivated Baccharis trimera. Journal of the Brazilian Chemical Society, 18(5), 990-997.

Simões-Pires, C. A., Debenedetti, S., Spegazzini, E., Mentz, L. A., Matzenbacher, N. I., Limberger, R. P., \& Henriques, A. T. (2005). Investigation of the essential oil from eight species of Baccharis belonging to sect. Caulopterae (Asteraceae, Astereae): a taxonomic approach. Plant Systematics and Evolution, 253, 23-32.

Sobrinho, C. A. N., Souza, E. B., Rocha, M. F. G., Albuquerque, M. R. J. R., Bandeira, P. N., Santos, H. S., Cavalcante, C. S. P., ..., \& Fontenelle, R. O. S. (2016). Chemical composition, antioxidant, antifungal and hemolytic activities of essential oil from
Baccharis trinervis (Lam.) Pers. (Asteraceae). Industrial Crops and Products, 84,108-115. http://dx.doi. org/10.1016/j.indcrop.2016.01.051

Sørensen, N. A. (1977). Polyacetylenes and conservatism of chemical characters in the Compositae. In V. H. Heywood, J. B. Harborne, \& B. L. Turner (Eds.). The biology and chemistry of the Compositae (Vol. 1, pp. 385-409). London: Academic Press.

van den Dool, H. \& Kratz, P. D. (1963). A generalization of the retention index system including linear temperature programmed gas-liquid partition chromatography. Journal of Chromatography A, 11, 463-471.

Vannini, A. B., Santos, T. G., Fleming, A. C., Purnhagen, L. R. P., Lourenco, L. A., Butzke, E. T. B., Kempt, M., ..., \& Steindel, M. (2012). Chemical characterization and antimicrobial evaluation of the essential oils from Baccharis uncinella D.C. and Baccharis semiserrata D.C. (Asteraceae). Journal of Essential Oil Research, 24(6), 547-554.

Verdi, L. G, Brighente, I. M. C., \& Pizzolatti, M. G. (2005). Gênero Baccharis (Asteraceae): aspectos químicos, económicos e biológicos. Química Nova, 28(1), 85-94. http://dx.doi.org/10.1590/ S0100-40422005000100017

Zoghbi, M. G. B., Andrade, E. H. A., Santos, A. S., Silva, M. H. L., \& Maia, J. G. S. (1998). Essential oils of Lippia alba (Mills.) N. E. Br. growing in the Brazilian Amazon. Flavour and Fragrance Journal, 13(1), 47-48. 